\title{
Tratamiento de la anquilosis temporomandibular en niños con materiales aloplásticos.
}

\author{
Treatment of temporomandibular ankilosis in \\ children with alloplastic materials.
}

Francisco Mercado Montañez*†

RESUMEN

La anquilosis de la articulación temporomandibular en niños continúa siendo una patología presente a pesar de los avances médicos y sociales. El tratamiento de esta patología en niños tiene como objetivos restablecer la apertura bucal y mejorar la estética facial cuando se presentan hipoplasias o micrognatias asociadas. El uso de materiales aloplásticos para el tratamiento de la anquilosis temporomandibular en niños es evitar la reanquilosis y disminuir riesgos, molestias y costos que ocasionan la toma y aplicación de injertos, siendo utilizados con buenos resultados en niños en otras especialidades como la Traumatología y Ortopedia. Estos procedimientos pueden llevarse a cabo de manera segura y predecible. En este artículo se reportan dos casos de anquilosis temporomandibular en niños, tratados con materiales aloplásticos, llevados a cabo en la Unidad Médica de Alta Especialidad No. 71 del Instituto Mexicano del Seguro Social, Torreón, Coahuila, México, con un seguimiento de 11 y 16 años de postoperatorio, demostrando que se trata de una buena opción de tratamiento sin presentar alteraciones al crecimiento y desarrollo de los pacientes.

Palabras clave: Anquilosis temporomandibular, materiales aloplásticos, cóndilo mandibular, niños.

\section{ABSTRACT}

Temporomandibular ankilosis in children is pathology still present despite the medical and social advances. The treatment of this pathology in children aims to restore mouth opening and improve facial aesthetics when hypoplasia or micrognatia are present. The use of alloplastic materials to treat temporomandibular ankilosis in children is to prevent the re ankilosis and reduce discomfort, risks, and cost causing by the take and application of graft, alloplastic materials being used with good results in children in other specialties such as Traumatology and Orthopedics. These procedures can be made safely and predictably. This article describes two cases of temporomandibular ankilosis in children, treated with alloplastic materials, carried out in the Medical Unit of High Specialty, number 71, of the Mexican Institute Social Security, Torreon, Coahuila, Mexico, with follow up of cases 11 and 16 years of postoperative, prove that is a good option of treatment, without presenting any alterations in growth and development of patients.

Keywords: Temporomandibular ankilosis, alloplastic materials, mandibular condyle, children.

\section{INTRODUCCIÓN}

L a anquilosis de la articulación temporomandibular en niños sigue siendo un problema de salud en dicha población, pese a los avances médicos y sociales. El factor etiológico de esta patología en niños son las fracturas del cóndilo mandibular durante la fase de crecimiento y desarrollo.

La anquilosis temporomandibular trae como resultado trastornos físicos, psicológicos y sociales como son la pérdida de la movilidad mandibular, dificultad o imposibilidad para masticar los alimentos con trastornos

\footnotetext{
* In memoriam: El Dr. Francisco Mercado Montañez, Cirujano Maxilofacial de la Unidad Médica de Alta Especialidad, IMSS, falleció el día 3 de diciembre de 2020. QEPD.

Recibido: 29 de julio de 2019. Aceptado: 18 de julio de 2021.

Citar como: Mercado MF. Tratamiento de la anquilosis temporomandibular en niños con materiales aloplásticos. Rev ADM. 2021 ; 78 (5): $291-296$. https://dx.doi.org/10.35366/102038
} 
digestivos, aseo bucal deficiente con caries o enfermedad periodontal de complicado tratamiento dental, dificultad para hablar o en casos extremos para respirar, detención del crecimiento y desarrollo mandibular que puede ser uni- o bilateral que deriva en deformidades faciales como hipoplasia mandibular o micrognatia. El tratamiento de esta patología implica procedimientos quirúrgicos poco frecuentes, de alto riesgo, costo elevado, y que se realizan en limitados centros hospitalarios, lo que convierte a esta alteración en un problema de salud social.

El tratamiento de la anquilosis temporomandibular ha sido bien documentado y reportado en múltiples artículos y libros referentes a esta patología, estableciéndose protocolos encaminados a restablecer la movilidad mandibular y restaurar la anatomía articular, a la vez de favorecer el desarrollo y crecimiento de las estructuras óseas dañadas. ${ }^{1}$ Para tales propósitos, los protocolos establecidos en niños con esta patología han sido la eliminación quirúrgica de los segmentos óseos fusionados, restaurando la articulación con la interposición de tejido autólogo tal como injerto de costilla, o fascia de músculo temporal entre el hueso temporal y rama mandibular, ${ }^{2}$ reportándose resultados adecuados para la apertura bucal en unos casos, sin resolver el problema de la falta de crecimiento, o la recidiva de la anquilosis a mediano plazo en otros, habiendo necesidad de realizar múltiples cirugías

Figura 1:

En la exploración clínica se aprecia

nula apertura

bucal, aspecto

facial con mínima

alteración estética

y micrognatia

mandibular.

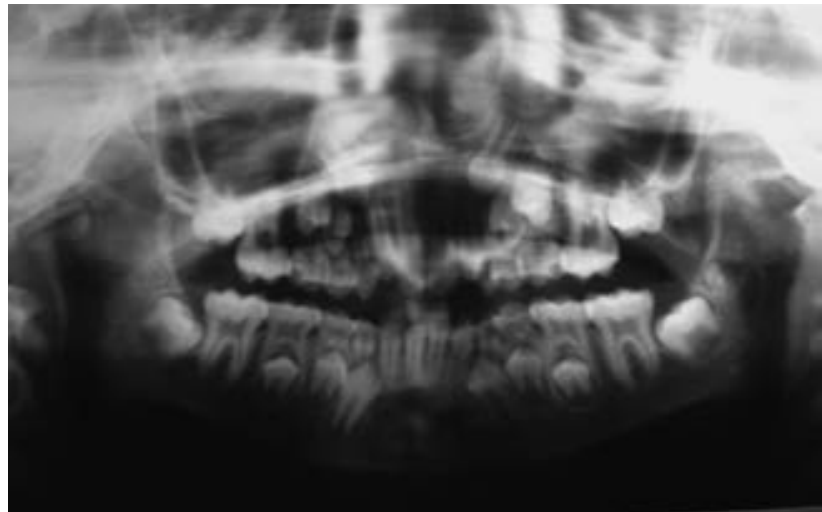

Figura 2: Radiografía panorámica inicial que muestra anquilosis temporomandibular bilateral.

que aumentan la morbilidad. ${ }^{3}$ Siempre se ha considerado tema de controversia el uso de materiales aloplásticos en pacientes en crecimiento y desarrollo como tratamiento de la anquilosis, argumentando el posible daño que se pueda ocasionar al crecimiento y desarrollo de la mandíbula, siendo que dicho daño ya está ocasionado al momento de que se lesiona el área articular. Algunos artículos recientes mencionan el uso de prótesis aloplásticas en paciente en desarroIlo con un periodo de crecimiento residual corto. ${ }^{4,5}$ En ortopedia, el uso de materiales aloplásticos en niños ha sido ampliamente documentado con buenos resultados. ${ }^{6}$

En este artículo se presentan dos casos de manejo de anquilosis temporomandibular en pacientes en periodo de crecimiento con materiales aloplásticos, siete y nueve años de edad, con un seguimiento a largo plazo de 11 y 18 años respectivamente, sin complicaciones qué reportar, no encontrando en la bibliografía casos de manejo de prótesis metálicas en niños para tratamiento de la anquilosis temporomandibular.

\section{MATERIAL Y MÉTODOS}

\section{Caso 1}

Se trata de paciente masculino de siete años de edad atendido en la Unidad Médica de Alta Especialidad (UMAE) No. 71 del Instituto Mexicano del Seguro Social, en la ciudad de Torreón, Coahuila, México. Acude por presentar imposibilidad de la apertura bucal desde la edad de cinco años, teniendo como único antecedente de importancia traumatismo facial 
al estar jugando y caer de su propia altura, evolucionando con progresiva limitación de apertura bucal hasta no poder abrirla en lo absoluto. A la exploración física, nula apertura bucal, aspecto facial con mínima alteración estética, micrognatia mandibular sin impacto estético importante (Figura 1). La radiografía inicial muestra anquilosis temporomandibular bilateral (Figura 2). Bajo anestesia general, intubación nasal con fibroscopio, se realiza liberación bilateral de anquilosis temporomandibular eliminando fragmentos óseos anquilosados y colocando prótesis metálicas de cavidad glenoidea de aleación cromo, cobalto, molibdeno (Figura 3), logrando adecuada apertura bucal sin complicaciones qué comentar (Figura 4). A la edad de 11 años se colocan prótesis metálicas de cóndilo de manera bilateral del mismo metal y mentoplastia de avance. Radiografía del paciente a la edad de 23 años (Figura 5).

A la edad de 25 años se realiza elevación de segmento anterior maxilar para corrección de exceso maxilar vertical sin complicaciones. Los abordajes quirúrgicos fueron preauricular bilateral, y submandibulares para la colocación de las prótesis, las cuales se fijan a hueso con tornillos de titanio, para la mentoplastia se utiliza abordaje en fondo de saco vestibular inferior. Para la elevación de la premaxila se utiliza abordaje en fondo de saco superior anterior. Apertura bucal y aspecto facial adecuados (Figura 6).

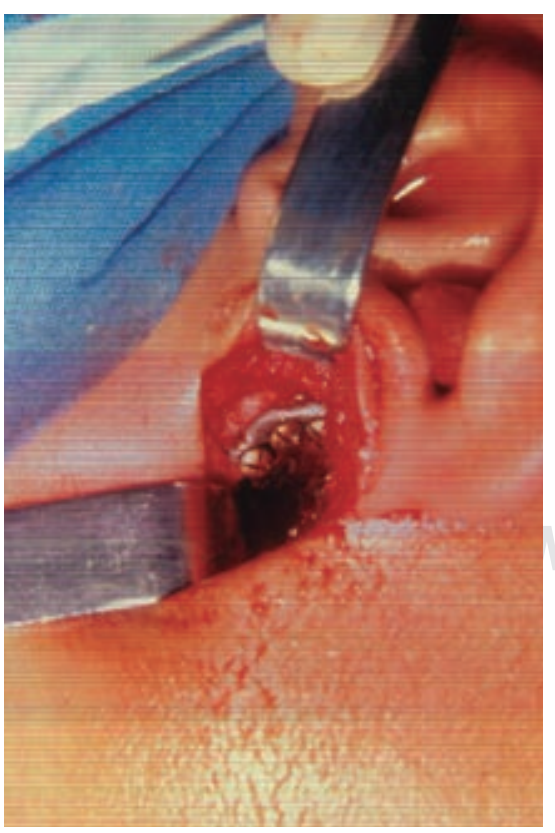

Figura 3:

Liberación quirúrgica bilateral de la anquilosis temporomandibular colocando prótesis metálicas de cavidad glenoidea.

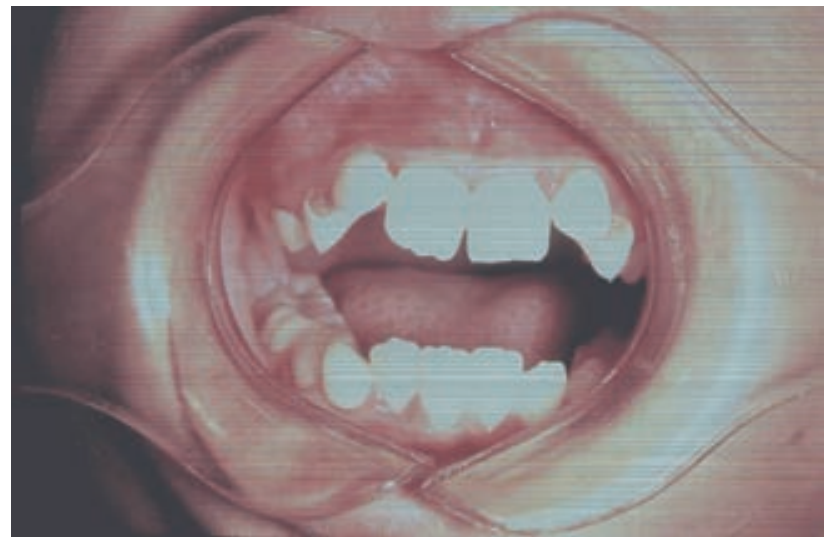

Figura 4: Después de la liberación bilateral se ha logrado sin complicaciones una adecuada apertura bucal.

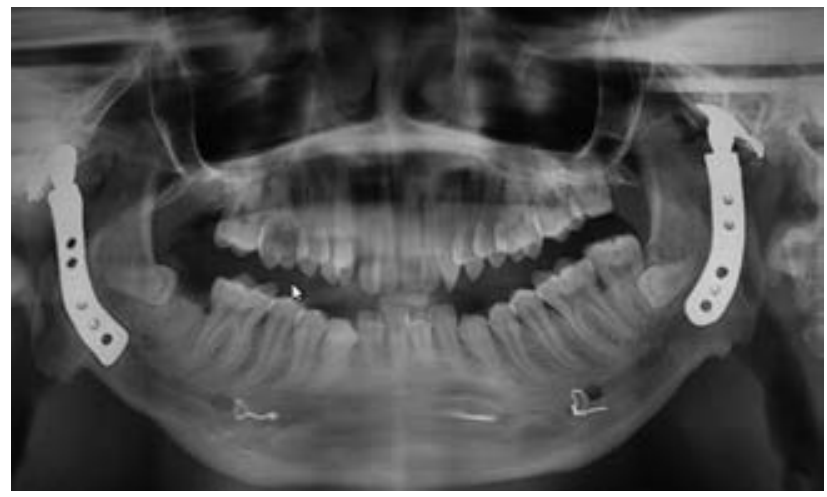

Figura 5: Paciente a la edad de 23 años con prótesis metálicas bilaterales del cóndilo y mentoplastia de avance. Radiografía muestra a 12 años de seguimiento.

\section{Caso 2}

Masculino de nueve años de edad intervenido quirúrgicamente en el año 2005 por presentar anquilosis temporomandibular derecha posterior a trauma facial al caer y golpearse la mandíbula (Figura 7). Nula apertura bucal, sin defecto facial importante (Figura 8). Bajo anestesia general intubación nasal con fibroscopio, se realiza liberación de anquilosis con colocación de prótesis articular metálica de titanio lado derecho, evolucionando a la fecha sin rechazo de prótesis, con excelente apertura bucal y estética facial (Figura 9). Presenta desde la primera intervención quirúrgica, ligera parálisis palpebral, que ha ido mejorando con los años. En el año 2016, se realiza mentoplastia de avance para 
mejorar la estética facial, se programa para extracción quirúrgica de segundo y tercer molares retenidos. A la fecha sin complicaciones qué reportar en su crecimiento y desarrollo (Figura 10).

\section{RESULTADOS}

El uso de prótesis metálicas para el tratamiento de la anquilosis temporomandibular en niños ha dado buenos resultados en esta unidad médica, consiguiendo los dos objetivos básicos, que el paciente abra la boca, y evitar nueva anquilosis. Dicho método se ha realizado desde hace más de 20 años con buenos resultados sin que se hayan presentado complicaciones o daños referentes a la falta de desarrollo de las estructuras faciales, o rechazo del material, siendo el manejo de estas prótesis aloplásticas en niños un método sólo utilizado en esta unidad, reportando dos casos del uso de las mismas de largo plazo. Las prótesis articulares metálicas utilizadas en niños tienen el mismo efecto benéfico que las utilizadas en adultos, evitando la morbilidad y costos que ocasiona la toma y aplicación de injertos.

\section{DISCUSIÓN}

La anquilosis temporomandibular continúa siendo una patología presente en la población infantil una vez que existe siempre el riego de lesión traumática a las articulaciones. El tratamiento de esta patología en niños ha sido la eliminación quirúrgica del o los segmentos anquilosados y

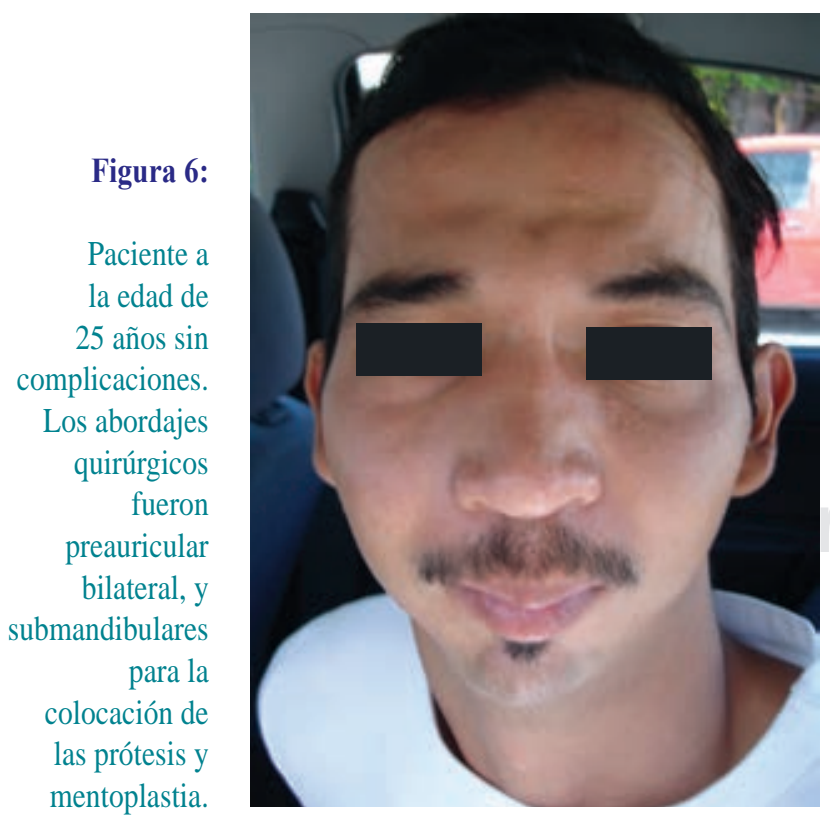

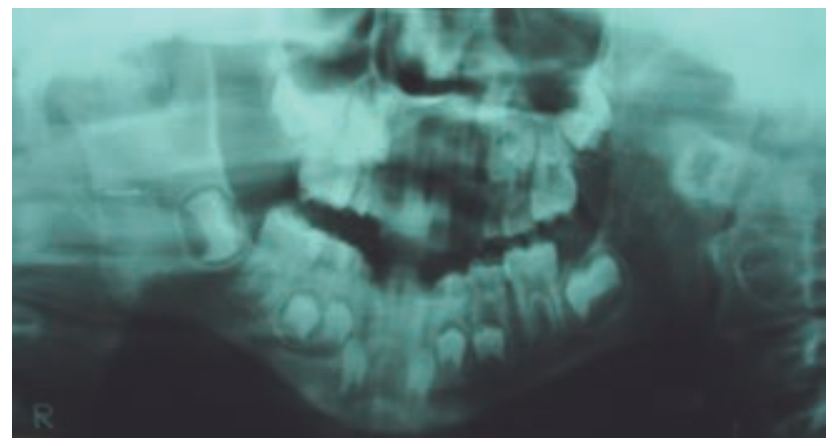

Figura 7: Menor de 3 años que presenta anquilosis temporomandibular derecha posterior a trauma facial al caer y golpearse la mandíbula.

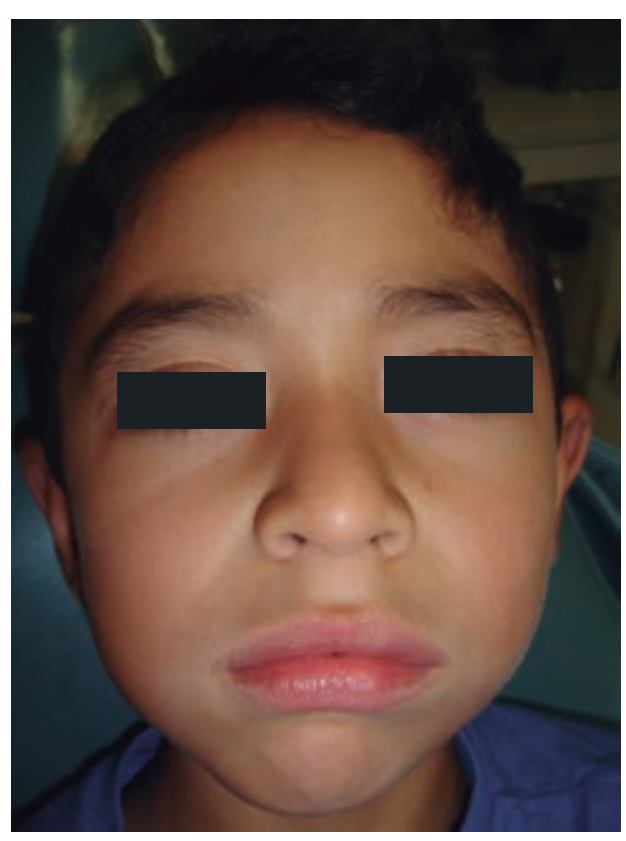

Figura 8: Paciente masculino a los nueve años de edad intervenido quirúrgicamente por presentar anquilosis temporomandibular derecha, sin defecto facial importante.

la colocación de injertos autólogos para favorecer el crecimiento mandibular detenido por el daño a los centros de crecimiento. ${ }^{7}$ Tales procedimientos conllevan la necesidad de realizar múltiples procedimientos quirúrgicos de difícil realización, mayores molestias y costos elevados. El uso de materiales aloplásticos en niños para el tratamiento de diversas patologías en el área de la traumatología y ortopedia ha sido muy documentado, ${ }^{8} \sin$ reporte de complicaciones que contraindiquen su utilización en este sector de la población. Su uso en el tratamiento de la anquilosis tempo- 
romandibular en niños ha sido muy poco documentado, ${ }^{9}$ siendo aún su utilización un tema de controversia, con el argumento de un posible daño a los procesos naturales del crecimiento y desarrollo facial, olvidando que dicho daño se encuentra ya presente una vez desarrollada esta patología. En este artículo, se reportan dos casos de uso de materiales aloplásticos para el tratamiento de la anquilosis en niños, con seguimiento a largo plazo, utilizando una aleación de cromo, cobalto, en uno de ellos, y titanio en otro, metales que desde hace muchos años se han utilizado en diferentes áreas de la cirugía y odontología, y que no ocasionan daño a las estructuras anatómicas más allá de las posibles complicaciones inherentes a cualquier procedimiento quirúrgico y odontológico. ${ }^{10}$ La fijación, en el caso del uso de cavidades glenoideas de aleación cromo-cobalto, se realizó con tornillos de titanio, ya que dicho metal tiene la característica de óseo integrarse, lo que le confiere estabilidad a las prótesis a largo plazo, para su fijación en cráneo, nos basamos en tomografías computarizadas para determinar el tamaño de los tornillos, evitando el posible daño a estructuras craneales. En estos casos no se observaron alteraciones desfavorables en el crecimiento y desarrollo de las estructuras craneofaciales, en el paciente con prótesis de cóndilo de titanio se ob-

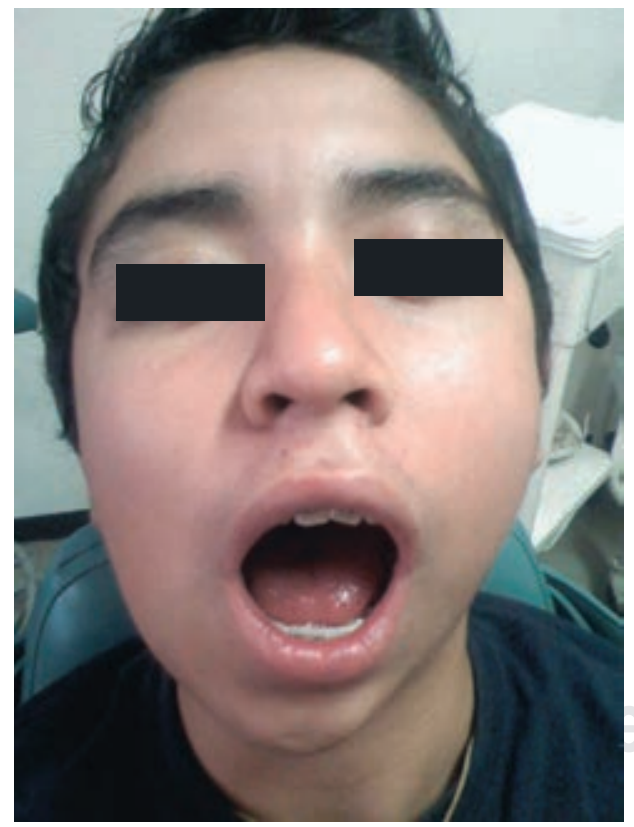

Figura 9: Paciente con liberación de anquilosis con colocación de prótesis articular evolucionando sin rechazo de prótesis, con excelente apertura bucal y estética facial, desde la primera intervención quirúrgica, presentó ligera parálisis palpebral, que ha ido mejorando.

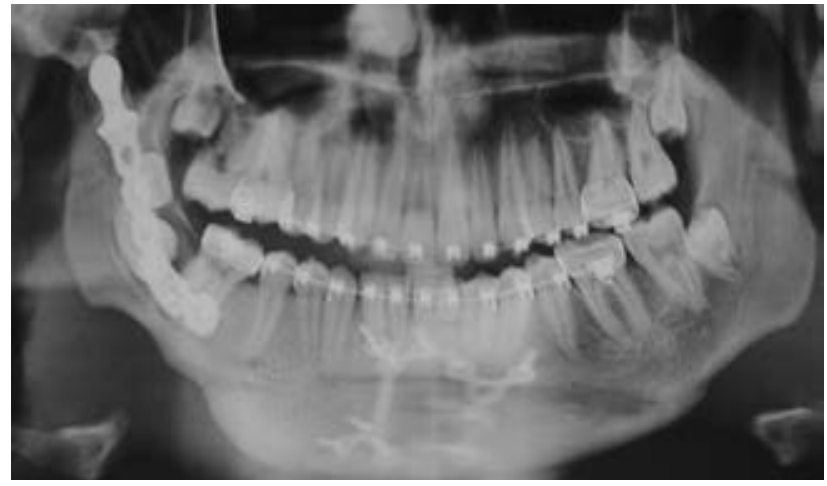

Figura 10: Radiografía de control donde se aprecia la prótesis de cóndilo y la mentoplastia de avance sin complicaciones en su crecimiento y desarrollo.

servó un adecuado crecimiento espontáneo de la rama mandibular afectada, situación poco común pero que ha sido reportada con el uso del titanio en reconstrucciones mandibulares. El manejo médico postoperatorio de los pacientes fue con antibioticoterapia a base de penicilina sódica cristalina a razón de 2 millones vía intravenosa cada ocho horas, por espacio de cinco días, antiinflamatorios tipo dexametasona $4 \mathrm{mg}$ cada 12 horas por tres días, y paracetamol $5 \mathrm{~mL}$ vía oral sólo por razón necesaria, no siendo el dolor una característica importante presente en estas intervenciones.

El tratamiento de la anquilosis temporomandibular en niños representa un difícil reto a resolver, ya que se trata de pacientes que se encuentran en periodo de crecimiento y desarrollo de sus estructuras anatómicas, fisiológicas y psicológicas, por lo que cualquier procedimiento a realizar incide directamente en estos aspectos. La toma y aplicación de injertos autólogos para el tratamiento de la anquilosis aumenta la cantidad de procedimientos quirúrgicos a realizar en los pacientes, los riesgos, molestias, y costos que de éstos se derivan, así como las posibles complicaciones con el uso de materiales aloplásticos para el manejo de esta patología en niños, de los cuales reportamos dos casos con seguimiento a largo plazo en este artículo.

\section{CONCLUSIONES}

Hemos podido comprobar que, de llevarse a cabo el procedimiento de manera adecuada, se evitan cirugías de toma de injertos, eliminando las molestias y posibles complicaciones que éstas conllevan, el postoperatorio inmediato presenta muy pocas molestias, los casos 
abren la boca de manera inmediata y pueden iniciar su función masticatoria sin restricción alguna, lo que favorece su nutrición, mejorando considerablemente su autoestima, además disminuyen considerablemente los costos, riesgos e impacto psicológico derivado de múltiples cirugías. No se observaron daños a estructuras anatómicas que alteraran el crecimiento y desarrollo craneofacial. Las prótesis han sido bien aceptadas, sin datos de rechazo a los 11 y 18 años de postoperatorio, siendo una muy buena opción de tratamiento para estos casos en nuestro hospital.

\section{REFERENCIAS}

1. Kaban LB, Bouchard C, Troulis MJ. A protocol for management of temporomandibular joint ankylosis in children. J Oral Maxillofac Surg. 2009; 67 (9): 1966-1978.

2. Wolford LM, Cottrell DA, Henry C. Sternoclavicular grafts for temporomandibular joint reconstruction. J Oral Maxillofac Surg. 1994; 52 (2): 119-128; discussion 128-129.
3. Guyuron B, Lasa Cl Jr. Unpredictable growth pattern of costochondral graft. Plast Reconstr Surg. 1992; 90 (5): 880-886; discussion 887-889.

4. Mercuri LG, Swift JQ. Considerations for the use of alloplastic temporomandibular joint replacement in the growing patient. J Oral Maxillofac Surg. 2009; 67 (9): 1979-1990.

5. Mercuri LG. The use of alloplastic prostheses for temporomandibular joint reconstruction. J Oral Maxillofac Surg. 2000; 58 (1): 70-75.

6. Carpenter EB. Resection of the proximal third of the femur for chondrosarcoma: replacement with a metallic prosthesis. A note after fifteen years of follow-up. J Bone Joint Surg Am. 1987; 69 (2): 279-281.

7. Broggi Ángulo OA, Cabrera Gómez EA, La Torre Caballero M, Oré Acevedo JF. Manejo contemporáneo de la anquilosis de la articulación temporomandibular en el niño y el adolescente: reporte de casos. Acta Méd Peruana. 2013; 30 (2): 86-91.

8. Singsen BH, Isaacson AS, Bernstein BH, Patzakis MJ, Kornreich HK, King KK et al. Total hip replacement in children with arthritis. Arthritis Rheum. 1978; 21 (4): 401-406.

9. Mercado MF, González AC. Reemplazo protésico de la articulación temporomandibular. Rev Mex Cir Buc Maxilofac. 2007; 3 (7): 92-96.

10. Mercado-Montañez F, Almanza PJ. Distracción ósea mandibular: reporte de 5 casos. Rev Mex Cir Bucal Max. 2008; 4 (9): 37-42. 